\title{
Structure and function of rat lymph nodes*
}

\author{
Osamu Ohtani and Yuko Ohtani
}

Department of Anatomy, Faculty of Medicine and Graduate School of Medicine and Pharmaceutical Sciences, University of Toyama, Toyama, Japan

Summary. The lymph node comprises a critical crossroad for encounters between antigen presenting cells, antigens from lymph, and lymphocytes recruited into lymph nodes from the blood. The node consists of spaces lined with lymphatic endothelial cells and parenchyma. The former spaces can be divided into the subcapsular sinuses, lymphatic labyrinths in the deep cortex, intermediate sinuses, and medullary sinuses. The sponge-like framework of the node parenchyma is composed of collagen fibers invested with reticular cells. The parenchyma can be divided into the cortex, deep cortex, and medullary cord. Lymphocytes migrate from the node parenchyma into the lymphatic labyrinths in the deep cortex. Close to the labyrinths are high endothelial venules (HEVs), through which circulating lymphocytes enter the node parenchyma. HEVs strongly express Aquaporin-1, suggesting that HEVs are involved in the net absorption of water, but not protein, from lymph coming through afferent lymphatics. Many LYVE-1 positive sinus reticular cells (i.e., lymphatic endothelial cells) with attached macrophages form a network within the lumen of the medullary sinuses. Fluids and migrating cells arriving at the node preferentially flow through the subcapsular

Received February 7, 2008

* This study was supported by a Grant-in-Aid for scientific research (Project No. 16390047) from the Japan Society for the Promotion of Science.

Address for correspondence: Prof. Osamu Ohtani, MD \& PhD, Department of Anatomy, Faculty of Medicine and Graduate School of Medicine \& Pharmaceutical Sciences, University of Toyama, Toyama, 930-0194 Japan

Tel: +81-76-434-7205, Fax: +81-76-434-5010

E-mail: osmotani@med.u-toyama.ac.jp sinuses, intermediate sinuses, and medullary sinuses in this order. Fluids and migrating cells may also enter the cortex through gaps in the floor of the subcapsular sinuses.

\section{Introduction}

The lymph node is a critical crossroad for encounters between: 1) antigen presenting cells; 2) antigenic substances from lymph, and 3) lymphocytes recruited into lymph nodes from the blood (Gretz et al., 1997). As the lymph node is densely packed with lymphocytes, dendritic cells, macrophages and more, it is quite difficult to show structures within the node in detail. Scanning electron microscopy, which has greatly developed from the latter half of the 20th century, has revealed the threedimensional ultrastructure of the lymph node (He, 1985; Ushiki, Ohtani, Abe, 1995). Recently discovered markers specific to lymphatic vessels have permitted disclosure of the lymphatic vascular system within the lymph node. These markers include prox-1 (Wigle and Oliver, 1999; Petrova et al., 2002), podoplanin (Wetterwald et al., 1996; Breiteneder-Geleff et al., 1999), LYVE-1 (Lymphatic Vessel Endothelial HA receptor 1; Banerji et al., 1999), VEGFR-3 (Kaipainen et al., 1995), a macrophage mannose receptor (Irjala et al., 2001), and CCL21 (Gunn et al., 1998).

It has been well established that the resorption of water and electrolytes, but not protein, occurs in the passage of lymph through lymph nodes; efferent lymph from a node typically has an increased protein concentration compared to afferent lymph arriving at the node (Renkin, 1986). Our previous study on rat mesenteric and mediastinal lymph nodes suggests that HEVs, which strongly express Aquaporin-1 (AQP-1) on both luminal and abluminal cell membranes, participate in the resorption of water.

In order to clarify the complex structure of lymph nodes, we have re-examined the node structure by 
immunohistochemistry to LYVE-1, which is integrated with our previous findings by scanning electron microscopy of intact tissues, immunohistochemistry to AQP-1, and tracer studies using fluorescent-labeled gelatin (Ohtani et al., 2003). In this study we used rabbit polyclonal antibodies to LYVE-1 (Abcam, California) and rabbit anti-AQP-1 antibodies (Chemicon International, Temecula).

\section{Lymph node structure and function}

In the lymph node, antigen presenting cells, antigenic substances from lymph, and lymphocytes recruited into lymph nodes from the blood converge to initiate primary immune responses (Gretz et al., 1997). The lymph node consists of two compartments: spaces lined with lymphatic endothelial cells, and parenchyma in which blood vessels travel. As the two compartments are intricate and penetrate each other, fluid and cellular pathways within the node remain to be determined precisely.

TRITC-gelatin injected into the small intestinal wall in rat fills the mesenteric lymph nodes, while that injected into the peritoneal cavity is rapidly transported to the mediastinal lymph nodes and fills their lymph pathways (Fig. 1). TRITC-gelatin was prepared according to the method introduced by Hashimoto and Kusakabe (1996). In brief, TRITC (Research Organics, Cleveland) was dissolved in a gelatin solution, and the resulting solution was dialyzed across a cellulose membrane (Seamless Cellulose Tubing ${ }^{\circledR}$, Wako Chemicals, Richmond) to remove free TRITC. TRITC-gelatin first appears in the subcapsular (or marginal) sinuses, which form a dense plexus (Fig. 1a). The gelatin then enters the space between the densely packed lymphocytes and cells of the cortex interstitium (Fig. 1b) and soon gathers into lymphatic vessels in the deep cortex and medullary sinuses (Fig. 1c, d). This finding indicates that macromolecules can freely enter the node cortex. There are occasional intermediate sinuses, which intervene between the subcapsular sinus and the medullary sinuses. Many relatively fine lymphatic vessels of less than $100 \mu \mathrm{m}$ in diameter originate from beneath the germinal centers (Fig. 1d, e). They exhibit several branchings and re-anastomoses to form a series of plexuses referred to as lymphatic labyrinths (He, 1985) in the deep cortex. These plexuses connect with the medullary sinuses, which are large, irregular, tortuous channels that repeatedly branch and anastomose (Fig. $1 \mathrm{c}, \mathrm{e})$. At the hilus, the medullary sinuses converge into the efferent lymphatic vessels of the node. Blood vessels course in the medullary cords which are themselves surrounded by the medullary sinuses (Fig. 1f).
Our scanning electron microscopic observation showed the three-dimensional organization of the lymph nodes (Ushiki, Ohtani, Abe, 1995; Ohtani et al., 2003) (Fig. 2). Many collagen trabecular fibers, invested by sinus lining cells (or retothelial cells), traverse the subcapsular sinus (Fig. 2a). The subcapsular sinus continues to the intermediate sinus which in turn connects with the medullary sinuses, though the intermediate sinuses are only occasionally found in the rat mesenteric lymph nodes. Flat sinus lining cells with their interrupted basement membrane line the wall of the subcapsular sinus adjacent to the cortical interstitium of the node, but this wall has many pores continuous with interstitial spaces of the cortex between the resident lymphocytes of the node (Ushiki, Ohtani, Abe, 1995) (Fig. 2a). The subcapsular and intermediate sinuses are lined with LYVE-1 positive endothelial cells (Fig. 3a). In the deep cortex, there are lymphatic labyrinths (He, 1985), with their lumen densely packed with lymphocytes. When we sliced the rat mesenteric lymph nodes with a vibratome, many free cells near the tissue surfaces were removed, thereby showing the lumens of lymphatic vessels with scattered lymphocytes, using scanning electron microscopy (Fig. $2 \mathrm{~b}, \mathrm{c})$. Few macrophages can be observed within the lymphatic labyrinths. The lymphatic labyrinth is lined with LYVE-1 positive lymphatic endothelial cells which are supported by very few intraluminal sinus reticular cells (Fig. 3b). Many lymphocytes are seen partly emergent from the endothelial cells when observed from the lumen of the lymphatic labyrinth, indicating that lymphocytes migrate from the node parenchyma into lymphatic labyrinths in the deep cortex (Fig. 2b). HEVs are frequently located close to the lymphatic labyrinths, the two structures being separated from each other only by a thin layer of interstitial space less than $10 \mu \mathrm{m}$ in thickness, which is usually packed with lymphocytes (Fig. 2c). HEVs are known to be the sites where lymphocytes migrate out of the blood vessels into the node parenchyma.

The lymphatic labyrinths merge into medullary sinuses, larger channels which are entirely lined with flat sinus lining cells that completely delimit the sinuses from medullary cords (Fig. 2d, 3c). Many sinus lining cells with attached macrophages form a network within the lumen of the medullary sinuses (Fig. 2d).

The sinus lining cells (also termed stellate cells, or retothelial cells) are in fact lymphatic endothelial cells similar to those lining the subcapsular sinuses, intermediate sinuses and lymphatic labyrinths because they express LYVE-1, a marker specific to lymphatic endothelial cells. The sinus lining cells are linked by junctions termed "complexus adhaerentes" (Schmelz et al, 1994). Recently, Hämmerling et al (2006) showed 
a
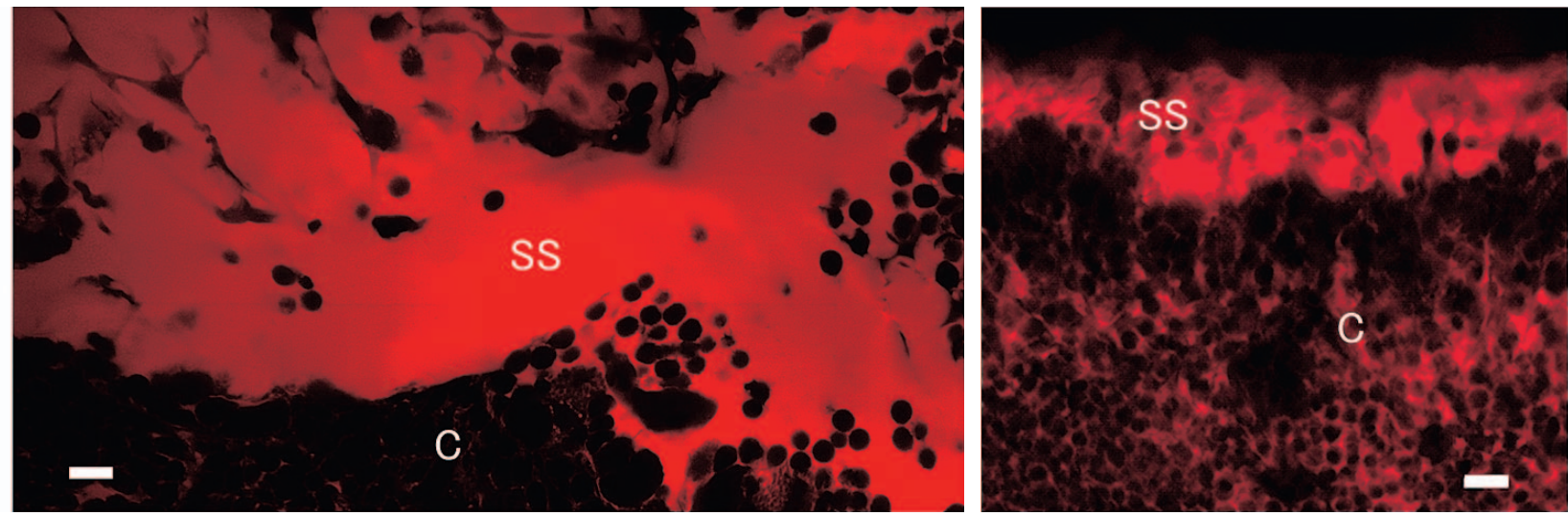

b
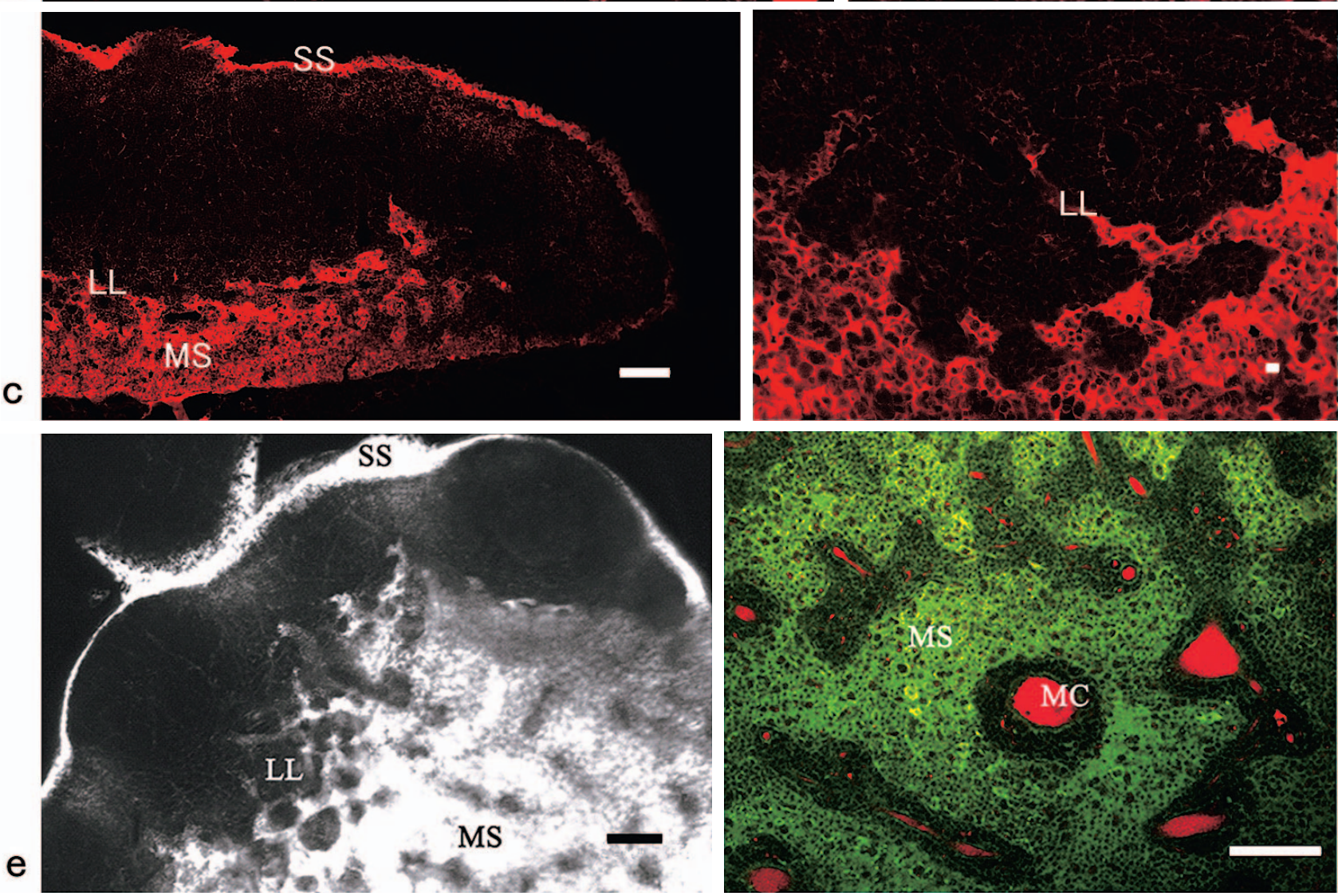

Fig. 1. Confocal micrographs of mediastinal (a-d) and mesenteric $(\mathbf{e}, \mathbf{f})$ nodes in the rat. a-d: TRITC- gelatin injected into the peritoneal cavity fills the subcapsular sinuses (SS) (a, b), and then enters the interstitial space of the cortex (C) (b). The gelatin drains into lymphatic labyrinths (LL) in the deep cortex, and finally into the medullary sinuses (MS) (c, d). e: The colored gelatin injected into the small intestinal wall fills the lymph pathways within the mesenteric lymph node. Note the lymphatic labyrinths in the deep cortex. f: Dual labeling of a lymph node by the injection of FITC-gelatin into the small intestinal wall and TRITC-gelatin into the blood vascular system shows the medullary sinuses (MS, green) are clearly demarcated from the medullary cords (MC) in which blood vessels (red) run. (b, e, f: from Ohtani et al., 2003) Scale bars $=10 \mu \mathrm{m}(\mathrm{a}, \mathrm{b}, \mathrm{d}), 100 \mu \mathrm{m}$ $(\mathrm{c}, \mathrm{e}, \mathrm{f})$ 

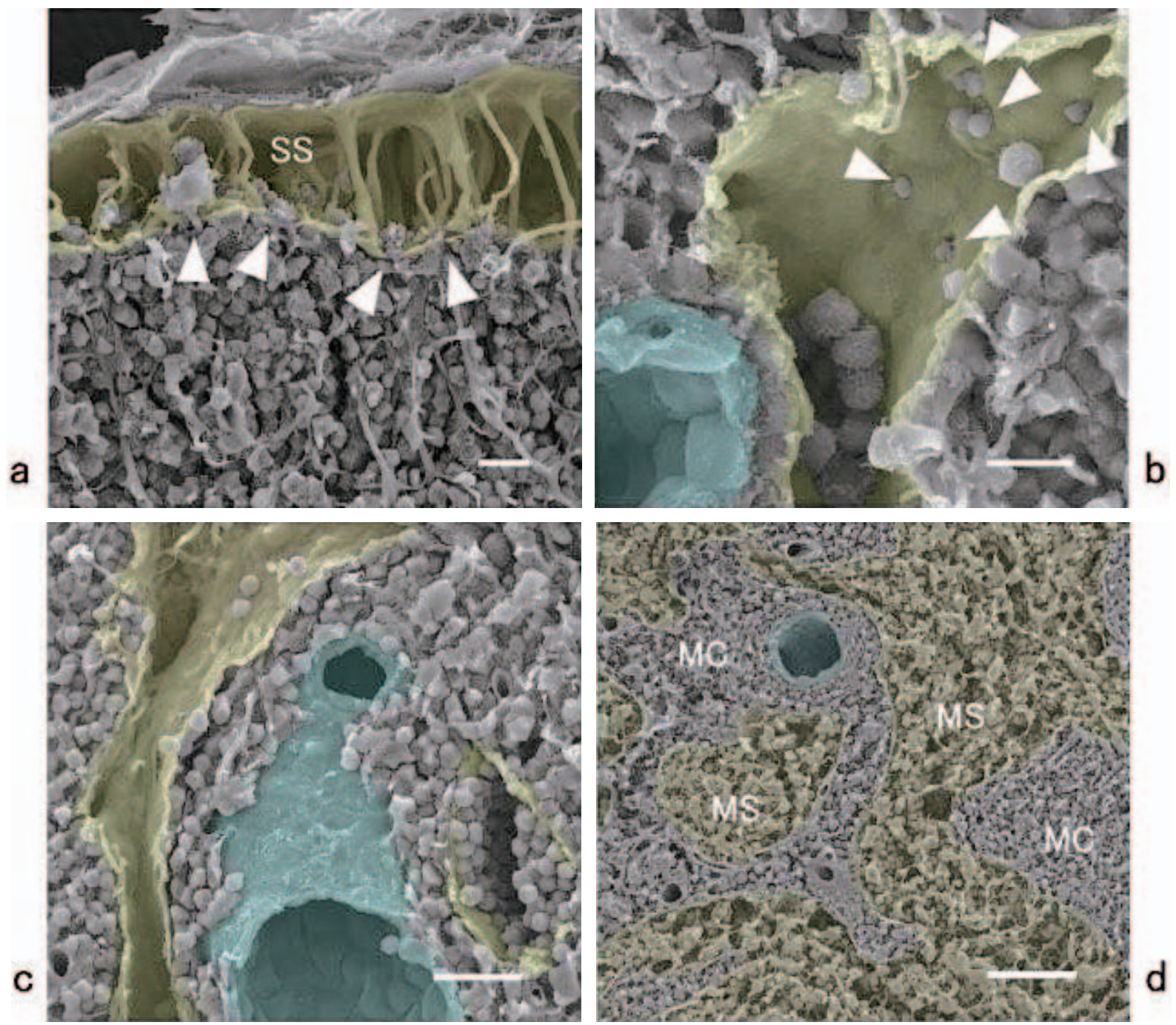

Fig. 2. Scanning electron micrographs of rat mesenteric lymph nodes. a: The floor of the subcapsular sinus (SS; yellow) possesses many pores (arrowheads) leading to the interstitial space of the cortex. b: A high power view of the lymphatic labyrinth (yellow) in the deep cortex shows lymphocytes (arrows) apparently emerging from the cortex into the lumen of the labyrinth. Note also a HEV (blue) located close to the labyrinth. c: The labyrinths (yellow) are separated from a HEV (blue) by only a thin layer of interstitium, which is largely occupied with loosely packed lymphocytes. d: The medullary sinuses (MS; yellow) with a network of sinus reticular cells are demarcated from the medullary cords only by a thin layer of lymphatic endothelial cells. There are dense networks of reticular (collagen) fibers invested with interstitial reticular cells as well as frequent blood vessels seen within the medullary cord. A HEV (blue) is seen in the medullary cord also. (Micrographs from Ohtani et al., 2003). Scale bars $=20 \mu \mathrm{m}$ (a), $10 \mu \mathrm{m}$ (b), $20 \mu \mathrm{m}$ (c), $50 \mu \mathrm{m}$ (d)

Fig. 3. Confocal micrographs of mesenteric lymph nodes from normal SD rats $(\mathbf{a}-\mathbf{d})$ and green rats $(\mathbf{e}-\mathbf{g})$. $\mathbf{a}-\mathbf{c}$ : The subcapsular sinus (SS), lymphatic labyrinths (LL), and medullary sinuses (MS) show a strong immunoreactivity to LYVE-1. Sinus reticular cells within the medullary sinuses are also LYVE-1 positive. d-g: Endothelial cells of the lymphatic labyrinths (LL) and blood vessels - especially of HEVs - show an immunoreactivity to AQP-1. AQP-1 is distributed on lymphatic endothelial and on both luminal and abluminal cell membranes of HEVs. A lymphatic labyrinth is located close to a HEV (e). Scale bars $=50 \mu \mathrm{m}$ (a), $25 \mu \mathrm{m}$ (b-d), $10 \mu \mathrm{m}$ (e), $5 \mu \mathrm{m}$ (f, g) 

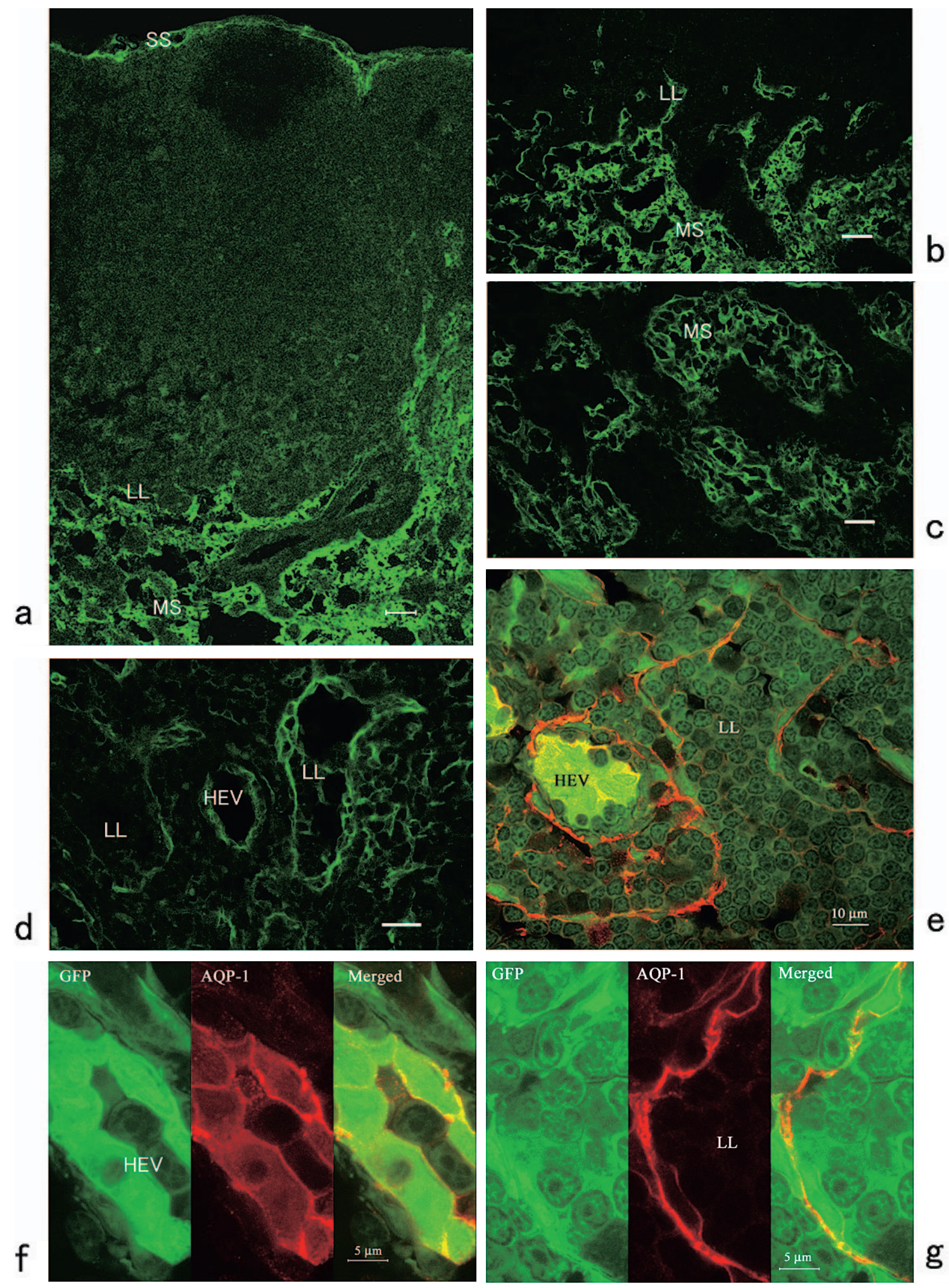

Fig. 3. Legend on the opposite page. 


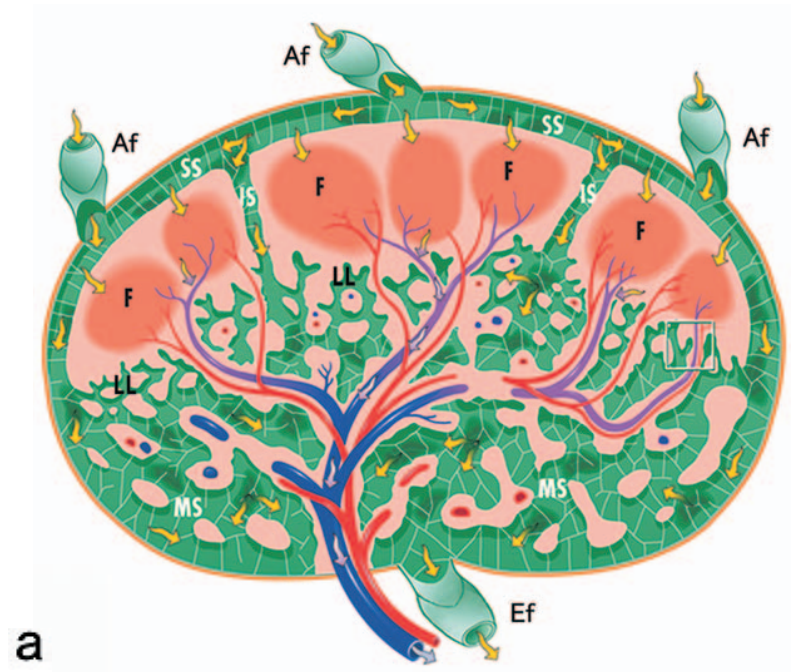

b
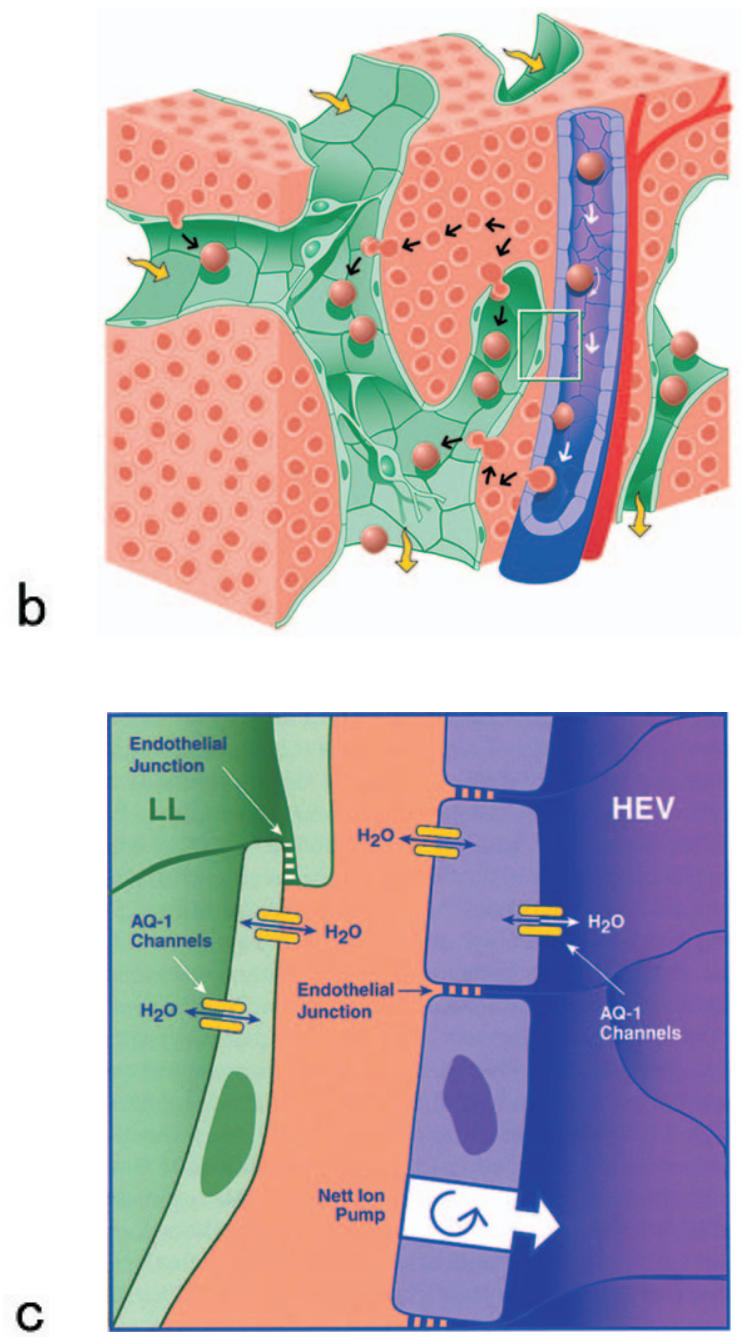

that the complexus adhaerentes of mammalian lymphatic endothelia comprises zonula adherens proteins (VEcadherin, $\alpha$ - and $\beta$-catenin, protein $\mathrm{p} 120^{\mathrm{ctn}}$, and afadin), desmosomal plaque components (desmoplakin and plakoglobin) and tight-junction proteins (claudin-5 and ZO-1).

In the interstitium of the medullary cords, there is a dense network of reticular fibers that is partly covered by the reticular cells; cut profiles of small blood vessels and larger HEVs are distributed in the parenchyma of the node, from the cortex to medullary cords (Fig. 2d).

It is well established that the resorption of water and electrolytes, but not protein, occurs in the passage of lymph through lymph nodes; efferent lymph of a node typically has an increased protein concentration compared with afferent lymph arriving at the node (see e.g. Fig 3 in Renkin, 1986). It is, however, not known what structures and/or mechanisms are involved in

Fig. 4. Schematic diagrams of the rat lymph node. a: This diagram shows an overview of lymphatic pathways (green), arteries (red) and veins (violet: HEVs; blue: ordinary vein) of the lymph node. Arrows indicate the direction of fluid flow. Cross lines in subcapsular sinuses (SS), intermediate sinus (IS), and medullary sinuses (MS) indicate the networks of intraluminal reticular cells (i.e., lymphatic endothelial cells). F: follicle, Af: afferent lymphatic vessels, Ef: efferent lymphatic vessels, LL: lymphatic labyrinth. b: Schematic of a closer view of the boxed area in $\mathbf{a}$. Thicker white arrows indicate a possible time sequence for the uppermost lymphocyte flowing into a $\mathrm{HEV}$, which subsequently rolls on (thiner white arrow), then adheres to the luminal surface, and finally penetrates through its endothelium to enter a lymph node parenchyma. Black arrows indicate that lymphocytes in the lymph node parenchyma move towards the lymphatic labyrinths and penetrate through their endothelium to enter the labyrinths. Yellow arrows indicate lymph fluid direction. c: Schematic of the boxed area in b. AQP-1 membrane channels in luminal and abluminal membranes of sinusoidal endothelial lining cells allow water flux between sinusoidal lymph (left) and the node interstitium (center) fluid compartments. AQP-1 membrane channels in luminal and abluminal membranes of the HEV endothelium permit water flux between the node interstitium and HEV plasma (right) fluid compartments. The postulated existence of a net ion pump (a polarized combination of ion pump(s), co-transpoters and/ or exchangers) would result in a net flux of ions from the node interstitium to HEV plasma, causing a near iso-osmotic water flux from the node interstitium to HEV plasma. (from Ohtani et al., 2003). 
the transfer of water and electrolytes from lymph to plasma in the lymph node. AQP-1 water channels are widely expressed in blood capillary and initial lymphatic endothelia and in epithelia involved in fluid transport (Agre et al., 1993; Nielsen et al., 1993; 1999; Devuyst et al., 1998). Our recent study using SD-Tg(CAG-EGFP) rats* (green rats; Amersham, Tokyo) shows that AQP-1 is distributed on lymphatic endothelial and on both luminal and abluminal cell membranes of HEVs as well as blood capillaries (Fig. 3d-g). As mentioned above, lymphatics in the node are closely located to HEVs. These seem to suggest that the nodal distribution of AQP-1 in HEV endothelium might be associated with a reported net lymph-to-plasma fluid flux in lymph nodes. Further studies are needed to obtain clear evidence for this fluid flux through the HEV endothelium. The lymph fluid and cellular pathways within the node discussed above are schematically shown in Figure 4.

In conclusion, the node is constitutes a critical crossroad for encounter between antigen presenting cells, antigens from lymph, and lymphocytes recruited into lymph nodes from the blood. The node can probably concentrate protein concentrations by the net absorption of water through HEV endothelium.

\section{Notes}

${ }^{*}$ The transgenic animals are covered by one or more of the following patents: (1) US 6172 188, EP 851874 , US 5958713 and EP 0815257, and under international patent application PCT/EP01/06848 and other pending and foreign applications; and (2) US 5625 048, US 5777 079, US 5804 387, US 5968 738, US 5994 077, US 6 054 321, US 6066 476, US 6077 707, US 6090 919, US 6124 128, US 6319 669, US 6,403,374, EP 0804457, EP 1104769, JP 3283523 and other pending and foreign patent applications.

\section{References}

Agre P, Preston GM, Smith BL, Jung JS, Raina S, Moon C, Guggino WB, Nielsen S: Aquaporin CHIP: the archetypal molecular water channel. Am J Physiol. 265: F463-476 (1993).

Banerji S, Ni J, Wang SX, Clasper S, Su J, Tammi R, Jones M, Jackson DG: LYVE-1, a new homologue of the CD44 glycoprotein, is a lymph-specific receptor for hyaluronan. J Cell Biol 144: 789-801 (1999).
Breiteneder-Geleff S, Soleiman A, Kowalski H, Horvat R, Amann G, Kriehuber E, Diem K, Weninger W, Tschachler E, Alitalo A, Kerjaschki D: Angiosarcomas express mixed endothelial phenotypes of blood and lymphatic capillaries. Podoplanin as a specific marker for lymphatic endothelium. Am J Pathol 154: 385-394 (1999).

Devuyst O, Nielsen S, Cosyns J-P, Smith BL, Agre P, Squifflet J-P, Pouthier D, Goffin E: Aquaporin-1 and endothelial nitric oxide synthase expression in capillary endothelia of human peritoneum. Am J Physiol 275: H234-242 (1998).

Gretz JE, Anderson AO, Shaw S: Cords, channels, corridors and conduits: critical architectural elements facilitating cell interactions in the lymph node cortex. Immunol Rev 156: 11-24 (1997).

Gunn MD, Tangemann K, Tam C, Cyster JG, Rosen SD, Williams LT: A chemokine expressed in lymphoid high endothelial venules promotes the adhesion and chemotaxis of naïve T lymphocytes. Proc Natl Acad Sci USA 95: 258-263 (1998).

Hämmerling B, Grund C, Boda-Heggemann J, Moll R, Franke WW: The complexus adhaerens of mammalian lymphatic endothelia revisited: a junction even more complex than hitherto thought. Cell Tissue Res 324: 5567 (2006).

He Y: Scanning electron microscope studies of the rat mesenteric lymph node with special reference to highendothelial venules and hitherto unknown lymphatic labyrinth. Arch Histol Jpn 48: 1-15 (1985).

Irjala H, Johansson EL, Grenman R, Alanen K, Salmi M, Jalkanen S: Mannose receptor is a novel ligand for Iselectin and mediates lymphocyte binding to lymphatic endothelium. J Exp Med 194: 1033-1042 (2001).

Kaipainen A, Korhonen J, Mustonen T, van Hinsbergh VWM, Fang G-H, Dumont D, Breitman M, Alitalo $\mathrm{K}$ : Expression of the fms-like tyrosine kinase 4 gene becomes restricted to lymphatic endothelium during development. Proc Natl Acad Sci USA 92: 3566-3570 (1995).

Hashimoto H, Kusakabe M: A method for threedimensional observation of capillary network. Cell Technol 15: 660-670 (1996).

Nielsen S, Kwon T-H, Christensen BM, Promeneur D, Frøkiaer J, Marples D: Physiology and pathophysiology of renal aquaporins. J Am Soc Nephrol 10: 647-663 (1999).

Nielsen S, Smith BL, Christensen EI, Agre P: Distribution of the aquaporin CHIP in secretory and resorptive epithelia and capillary endothelia. Proc Natl Acad Sci USA 90: 7275-7279 (1993). 
Ohtani O, Ohtani Y, Carati CJ, Gannon BJ: Fluid and cellular pathways of rat lymph nodes in relation to lymphatic labyrinths and Aquaporin-1 expression. Arch Histol Cytol 66: 261-272 (2003).

Petrova TV, Mäkinen T, Mäkelä TP, Saarela J, Virtanen I, Ferrell RE, Finegold DN, Kerjaschki D, Ylä-Herttuala S, Alitalo K: Lymphatic endothelial reprogramming of vascular endothelial cells by the prox-1 homeobox transcription factor. EMBO J 21:4593-4599 (2002).

Renkin EM.: Some consequences of capillary permeability to macromolecules: Starling's hypothesis reconsidered. Am J Physiol 250: H706-710 (1986).

Schmelz M, Moll R, Kuhn C, Franke WW: Complexus adhaerentes, a new group of desmoplakin-containing junctions in endothelial cells: II. Different types of lymphatic vessels. Diffentiation 57: 97-117 (1994).
Ushiki T, Ohtani O, Abe K: Scanning electron microscopic studies of reticular framework in the rat mesenteric lymph node. Anat Rec 241: 113-122 (1995). Wetterwald A, Hofstetter W, Cecchini MG, Lanske B, Wagner C, Fleisch H, Atkinson M: Characterization and cloning of the e 11 antigen, a marker expressed by rat osteoblasts and osteocytes. Bone 18: 125-132 (1996).

Wigle JT, Oliver G: Prox1 function is required for the development of the murine lymphatic system. Cell 98: 769-778 (1999). 